\title{
The Complex Multivariate Gaussian Distribution
}

by Robin K. S. Hankin

\begin{abstract}
Here I introduce package cmvnorm, a complex generalization of the mvtnorm package. A complex generalization of the Gaussian process is suggested and numerical results presented using the package. An application in the context of approximating the Weierstrass $\sigma$-function using a complex Gaussian process is given.
\end{abstract}

\section{Introduction}

Complex-valued random variables find applications in many areas of science such as signal processing (Kay, 1989), radio engineering (Ozarow, 1994), and atmospheric physics (Mandic et al., 2009). In this short paper I introduce cmvnorm (Hankin, 2015), a package for investigating one commonly encountered complex-valued probability distribution, the complex Gaussian.

The real multivariate Gaussian distribution is well supported in R by package mvtnorm (Genz et al., 2014), having density function

$$
f(\mathbf{x} ; \mathbf{m}, \Sigma)=\frac{e^{-\frac{1}{2}(\mathbf{x}-\mathbf{m})^{T} \Sigma^{-1}(\mathbf{x}-\mathbf{m})}}{\sqrt{|2 \pi \Sigma|}} \quad \mathbf{x} \in \mathbb{R}^{n},
$$

where $|M|$ denotes the determinant of matrix $M$. Here, $\mathbf{m}=\mathbb{E}[\mathbf{X}] \in \mathbb{R}^{n}$ is the mean vector and $\Sigma=$ $\mathbb{E}\left[(\mathbf{X}-\mathbf{m})(\mathbf{X}-\mathbf{m})^{T}\right]$ the covariance of random vector $\mathbf{X}$; we write $\mathbf{X} \sim \mathcal{N}_{n}(\mathbf{m}, \Sigma)$. One natural generalization would be to consider $\mathbf{Z} \sim \mathcal{N C}_{n}(\mathbf{m}, \Gamma)$, the complex multivariate Gaussian, with density function

$$
f(\mathbf{z} ; \mathbf{m}, \Gamma)=\frac{e^{-(\mathbf{z}-\mathbf{m})^{*} \Gamma^{-1}(\mathbf{z}-\mathbf{m})}}{|\pi \Gamma|} \quad \mathbf{z} \in \mathbb{C}^{n},
$$

where $\mathbf{z}^{*}$ denotes the Hermitian transpose of complex vector $\mathbf{z}$. Now $\mathbf{m} \in \mathbb{C}^{n}$ is the complex mean and $\Gamma=\mathbb{E}\left[(\mathbf{Z}-\mathbf{m})(\mathbf{Z}-\mathbf{m})^{*}\right]$ is the complex variance; $\Gamma$ is a Hermitian positive definite matrix. Note the simpler form of (2), essentially due to Gauss's integral operating more cleanly over the complex plane than the real line:

$$
\int_{\mathbb{C}} e^{-z^{*} z} d z=\int_{x \in \mathbb{R}} \int_{y \in \mathbb{R}} e^{-\left(x^{2}+y^{2}\right)} d x d y=\int_{\theta=0}^{2 \pi} \int_{r=0}^{\infty} e^{-r^{2}} r d r d \theta=\pi .
$$

A zero mean complex random vector $\mathbf{Z}$ is said to be circularly symmetric (Goodman, 1963) if $\mathbb{E}\left[\mathbf{Z Z} \mathbf{Z}^{T}\right]=0$, or equivalently $\mathbf{Z}$ and $e^{i \alpha} \mathbf{Z}$ have identical distributions for any $\alpha \in \mathbb{R}$. Equation (2) clearly has this property.

Most results from real multivariate analysis have a direct generalization to the complex case, as long as "transpose" is replaced by "Hermitian transpose". For example, $\mathbf{X} \sim \mathcal{N}_{n}(\mathbf{0}, \Sigma)$ implies $B \mathbf{X} \sim \mathcal{N}_{n}\left(\mathbf{0}, B^{T} \Sigma B\right)$ for any constant matrix $B \in \mathbb{R}^{m \times n}$, and analogously $\mathbf{Z} \sim \mathcal{N} \mathcal{C}_{n}(\mathbf{0}, \Gamma)$ implies $B \mathbf{Z} \sim \mathcal{N C}_{n}\left(\mathbf{0}, B^{*} \Gamma B\right), B \in \mathbb{C}^{m \times n}$. Similar generalizations operate for Schur complement methods on partitioned matrices.

Also, linear regression generalizes similarly. Specifically, consider $\mathbf{y} \in \mathbb{R}^{n}$. If $\mathbf{y}=X \boldsymbol{\beta}+\boldsymbol{\epsilon}$ where $X$ is a $n \times p$ design matrix, $\beta \in \mathbb{R}^{p}$ a vector of regression coefficients and $\epsilon \sim \mathcal{N}_{n}(0, \Sigma)$ is a vector of errors, then $\hat{\beta}=\left(X^{T} \Sigma^{-1} X\right)^{-1} X^{T} \Sigma^{-1} \mathbf{y}$ is the maximum likelihood estimator for $\beta$. The complex generalization is to write $\mathbf{z}=Z \boldsymbol{\beta}+\boldsymbol{\epsilon}, Z \in \mathbb{C}^{n \times p}, \boldsymbol{\beta} \in \mathbb{C}^{p}, \boldsymbol{\epsilon} \sim \mathcal{N C}_{n}(\mathbf{0}, \Gamma)$ which gives $\hat{\beta}=\left(Z^{*} \Gamma^{-1} Z\right)^{-1} Z^{*} \Gamma^{-1} \mathbf{z}$. Such considerations suggest a natural complex generalization of the Gaussian process.

This short vignette introduces the cmvnorm package which furnishes some functionality for the complex multivariate Gaussian distribution, and applies it in the context of a complex generalization of the emulator package (Hankin, 2005), which implements functionality for investigating (real) Gaussian processes. 


\section{The package in use}

Random complex vectors are generated using the $r$ cmvnorm() function, analogous to rmvnorm():

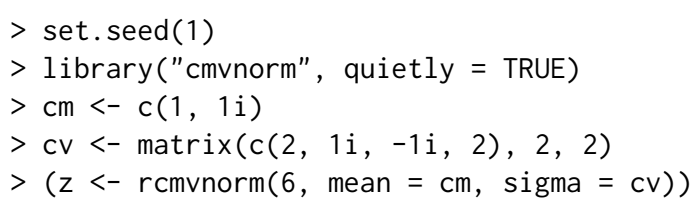

[,1] [,2]

$[1] \quad 0.9680986+,0.5525419 \mathrm{i} \quad 0.0165969+2.9770976 \mathrm{i}$

$[2] \quad 0.2044744-,1.4994889 \mathrm{i} \quad 1.8320765+0.8271259 \mathrm{i}$

$[3] 1.0739973+,0.2279914 \mathrm{i}-0.7967020+0.1736071 \mathrm{i}$

$[4] \quad 1.3171073-,0.9843313 i \quad 0.9257146+0.5524913 i$

$[5] 1.3537303-,0.8086236 \mathrm{i}-0.0571337+0.3935375 \mathrm{i}$

$[6] \quad 2.9751506-,0.1729231 \mathrm{i} \quad 0.3958585+3.3128439 \mathrm{i}$

Function dcmvnorm() returns the density according to (2):

$>\operatorname{dcmvnorm}(z, \mathrm{~cm}, \mathrm{cv})$

[1] $5.103754 \mathrm{e}-04 \quad 1.809636 \mathrm{e}-05 \quad 2.981718 \mathrm{e}-03 \quad 1.172242 \mathrm{e}-03 \quad 4.466836 \mathrm{e}-03 \quad 6.803356 \mathrm{e}-07$

So it is possible to determine a maximum likelihood estimate for the mean using direct numerical optimization

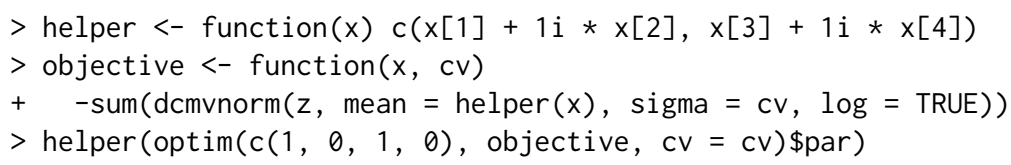

[1] $1.315409-0.447863 \mathrm{i} \quad 0.385704+1.372762 \mathrm{i}$

(helper functions are needed because optim() optimizes over $\mathbb{R}^{n}$ as opposed to $\mathbb{C}^{n}$ ). This shows reasonable agreement with the true value of the mean and indeed the analytic value of the MLE, specifically

$>\operatorname{colMeans}(z)$

[1] $1.315426-0.447472 \mathrm{i} \quad 0.386068+1.372784 \mathrm{i}$

\section{The Gaussian process}

In the context of the emulator, a (real) Gaussian process is usually defined as a random function $\eta: \mathbb{R}^{p} \longrightarrow \mathbb{R}$ which, for any set of points $\left\{\mathbf{x}_{1}, \ldots, \mathbf{x}_{n}\right\}$ in its domain $\mathcal{D}$ the random vector $\left\{\eta\left(\mathbf{x}_{1}\right)\right.$, $\left.\ldots, \eta\left(\mathbf{x}_{n}\right)\right\}$ is multivariate Gaussian.

It is convenient to specify $\mathbb{E}[\eta(\mathbf{x}) \mid \boldsymbol{\beta}]=h(\mathbf{x}) \boldsymbol{\beta}$, that is, the expectation of the process at point $\mathbf{x} \in \mathcal{D}$ conditional on the (unknown) vector of coefficients $\beta$. Here $h: \mathbb{R}^{p} \longrightarrow \mathbb{R}^{q}$ specifies the $q$ known regressor functions of $\mathbf{x}=\left(x_{1}, \ldots, x_{p}\right)^{T}$; a common choice is $h(\mathbf{x})=\left(1, x_{1}, \ldots, x_{p}\right)^{T}$ [giving $q=p+1$ ], but one is in principle free to choose any function of $\mathbf{x}$. One writes $H^{T}=\left(h\left(\mathbf{x}_{1}\right), \ldots, h\left(\mathbf{x}_{n}\right)\right)$ when considering the entire design matrix $X$; the $\mathrm{R}$ idiom is regressor.multi().

The covariance is typically given by

$$
\operatorname{cov}\left(\eta(\mathbf{x}), \eta\left(\mathbf{x}^{\prime}\right)\right)=V\left(\mathbf{x}-\mathbf{x}^{\prime}\right)
$$

where $V: \mathbb{R}^{n} \longrightarrow \mathbb{R}$ must be chosen so that the variance matrix of any finite set of observations is always positive-definite. Bochner's theorem (Feller, 1971, chapter XIX) shows that $V(\cdot)$ must be proportional to the characteristic function $(\mathrm{CF})$ of a symmetric probability Borel measure.

Oakley (1999) uses techniques which have clear complex analogues to show that the posterior mean of $\eta(\mathbf{x})$ is given by

$$
h(\mathbf{x})^{T} \boldsymbol{\beta}+\left(\operatorname{cov}\left(\mathbf{x}, \mathbf{x}_{1}\right), \ldots, \operatorname{cov}\left(\mathbf{x}, \mathbf{x}_{n}\right)\right)^{T} A^{-1}(\mathbf{y}-H \hat{\boldsymbol{\beta}}) .
$$


Here $A$ is an $n \times n$ matrix of correlations between the observations, $\sigma^{2} A_{i j}=\operatorname{cov}\left(\eta\left(\mathbf{x}_{i}\right), \eta\left(\mathbf{x}_{j}\right)\right)$ where $\sigma^{2}$ is an overall variance term; and $\hat{\boldsymbol{\beta}}=\left(X^{T} A^{-1} X\right)^{-1} X^{T} A^{-1} \mathbf{y}$ is the maximum likelihood estimator for $\beta$.

Equation (3) furnishes a cheap approximation to $\eta(\mathbf{x})$ and is known as the 'emulator'.

\section{Complex Gaussian processes}

The complex case is directly analogous, with $\eta: \mathbb{C}^{p} \longrightarrow \mathbb{C}$ and $\beta \in \mathbb{C}^{q}$. Writing $\operatorname{cov}\left(\eta\left(\mathbf{z}_{1}\right), \ldots, \eta\left(\mathbf{z}_{n}\right)\right)$ $=\Omega$, so that element $(i, j)$ of matrix $\Omega$ is $\operatorname{cov}\left(\eta\left(\mathbf{z}_{i}\right), \eta\left(\mathbf{z}_{j}\right)\right)$, we may relax the requirement that $\Omega$ be symmetric positive definite to requiring only Hermitian positive definiteness. This allows one to use the characteristic function of any, possibly non-symmetric, random variable $\Psi$ with density function $f: \mathbb{R}^{p} \longrightarrow \mathbb{R}$ and characteristic function $\phi:$

$$
\Omega_{i j}=\operatorname{cov}\left(\eta\left(\mathbf{z}_{i}\right), \eta\left(\mathbf{z}_{j}\right)\right)=\phi\left(\mathbf{z}_{i}-\mathbf{z}_{j}\right) .
$$

That $\Omega$ remains Hermitian positive definite may be shown by evaluating a quadratic form with it and arbitrary $\mathbf{w} \in \mathbb{C}^{n}$ and establishing that it is real and non-negative:

$$
\begin{aligned}
\mathbf{w}^{*} \Omega \mathbf{w} & =\sum_{i, j} \overline{\mathbf{w}_{i}} \operatorname{cov}\left(\eta\left(\mathbf{z}_{i}\right), \eta\left(\mathbf{z}_{j}\right)\right) \mathbf{w}_{j} \\
& =\sum_{i, j} \overline{\mathbf{w}_{i}} \phi\left(\mathbf{z}_{i}-\mathbf{z}_{j}\right) \mathbf{w}_{j} \\
& =\sum_{i, j} \overline{\mathbf{w}_{i}}\left[\int_{\mathbf{t} \in \mathrm{C}^{n}} e^{\mathrm{i} \operatorname{Re} \mathbf{t}^{*}\left(\mathbf{z}_{i}-\mathbf{z}_{j}\right)} f(\mathbf{t}) d \mathbf{t}\right] \mathbf{w}_{j} \\
& =\int_{\mathbf{t} \in \mathbb{C}^{n}}\left[\sum_{i, j} \overline{\mathbf{w}_{i}} e^{\mathrm{i} \operatorname{Re} \mathbf{t}^{*}\left(\mathbf{z}_{i}-\mathbf{z}_{j}\right)} \mathbf{w}_{j} f(\mathbf{t})\right] d \mathbf{t} \\
& =\int_{\mathbf{t} \in \mathbb{C}^{n}}\left[\sum_{i, j} \overline{\mathbf{w}_{i}} e^{\mathrm{i} \operatorname{Re}\left(\mathbf{t}^{*} \mathbf{z}_{i}\right)} \overline{\overline{\mathbf{w}_{j}}} e^{\mathrm{i} \operatorname{Re}\left(\mathbf{t}^{*} \mathbf{z}_{j}\right)} f(\mathbf{t})\right] d \mathbf{t} \\
& =\int_{\mathbf{t} \in \mathbb{C}^{n}}\left|\sum_{i} \overline{\mathbf{w}_{i}} e^{\mathrm{i} \operatorname{Re}\left(\mathbf{t}^{*} \mathbf{z}_{i}\right)}\right|^{2} f(\mathbf{t}) d \mathbf{t} \\
& \geqslant 0 .
\end{aligned}
$$

definition of quadratic form

covariance function is the $\mathrm{CF}$ of $\Psi$

$$
\text { definition of CF of } \Psi
$$

integration and summation commute

expand and rearrange

algebra

integral of sum of real positive functions

(This motivates the definition of the characteristic function of a complex multivariate random variable $\mathbf{Z}$ as $\left.\mathbb{E}\left[e^{i \operatorname{Re}\left(\mathbf{t}^{*} \mathbf{Z}\right)}\right]\right)$. Thus the covariance matrix is Hermitian positive definite: although its entries are not necessarily real, its eigenvalues are all nonnegative.

In the real case one typically chooses $\Psi$ to be a zero-mean Gaussian distribution; in the complex case one can use the complex multivariate distribution given in equation (2) which has characteristic function

$$
\exp \left(i \operatorname{Re}\left(\mathbf{t}^{*} \mathbf{m}\right)-\frac{1}{4} \mathbf{t}^{*} \Gamma \mathbf{t}\right)
$$

and following Hankin (2012) in writing $\mathfrak{B}=\Gamma / 4$, we can write the variance matrix as a product of a (real) scalar $\sigma^{2}$ term and

$$
c(\mathbf{t})=\exp \left(i \operatorname{Re}\left(\mathbf{t}^{*} \mathbf{m}\right)-\mathbf{t}^{*} \mathfrak{B} \mathbf{t}\right) .
$$

Thus the covariance matrix $\Omega$ is given by

$$
\Omega_{i j}=\operatorname{cov}\left(\eta\left(\mathbf{z}_{i}\right), \eta\left(\mathbf{z}_{j}\right)\right)=\sigma^{2} c\left(\mathbf{z}_{i}-\mathbf{z}_{j}\right) .
$$

In (6), $\mathfrak{B}$ has the same meaning as in conventional emulator techniques and controls the modulus of the covariance between $\eta(\mathbf{z})$ and $\eta\left(\mathbf{z}^{\prime}\right)$; $\mathbf{m}$ governs the phase.

Given the above, it seems to be reasonable to follow Oakley (1999) and admit only diagonal $\mathfrak{B}$; but now distributions with nonzero mean can be considered (compare the real case which requires 
a zero mean). A parametrization using diagonal $\mathfrak{B}$ and complex mean vector requires $3 p$ (real) hyperparameters; compare $2 p$ if $\mathbb{C}^{p}$ is identified with $\mathbb{R}^{2 p}$.

\section{Functions of several complex variables}

Analytic functions of several complex variables are an important and interesting class of objects; Krantz (1987) motivates and discusses the discipline. Formally, consider $f: \mathbb{C}^{n} \longrightarrow \mathbb{C}, n \geqslant 2$ and write $f\left(z_{1}, \ldots, z_{n}\right)$. Function $f$ is analytic if it satisfies the Cauchy-Riemann conditions in each variable separately, that is $\partial f / \partial \bar{z}_{j}=0,1 \leqslant j \leqslant n$.

Such an $f$ is continuous (due to a "non-trivial theorem of Hartogs") and continuously differentiable to arbitrarily high order. Krantz goes on to state some results which are startling if one's exposure to complex analysis is restricted to functions of a single variable: for example, any isolated singularity is removable.

\section{Numerical illustration of these ideas}

The natural definition of complex Gaussian processes above, together with the features of analytic functions of several complex variables, suggests that a complex emulation of analytic functions of several complex variables might be a useful technique.

The ideas presented above, and the cmvnorm package, can now be used to sample directly from an appropriate complex Gaussian distribution and estimate the roughness parameters:

$>$ val <- latin.hypercube $(40,2$, names $=c(" a ", " b ")$, complex $=$ TRUE $)$

$>\operatorname{head}(\mathrm{val})$

a $\quad$ b

$[1] \quad 0.7375+,0.2375 i \quad 0.2375+0.7125 i$

$[2] \quad 0.6875+,0.5875 \mathrm{i} \quad 0.1375+0.3375 \mathrm{i}$

$[3] \quad 0.4625+,0.5375 i \quad 0.9875+0.5875 i$

$[4] \quad 0.7875+,0.0625 i \quad 0.0625+0.7875 i$

$[5] \quad 0.3875+,0.0375 i \quad 0.5875+0.7625 i$

$[6] \quad 0.2125+,0.5625 i \quad 0.7625+0.9625 i$

(function latin.hypercube () is used to generate a random complex design matrix). We may now specify a variance matrix using simple values for the roughness hyperparameters $\mathfrak{B}=\left(\begin{array}{ll}1 & 0 \\ 0 & 2\end{array}\right)$ and $\mathbf{m}=$ $(1, i)^{T}$ :

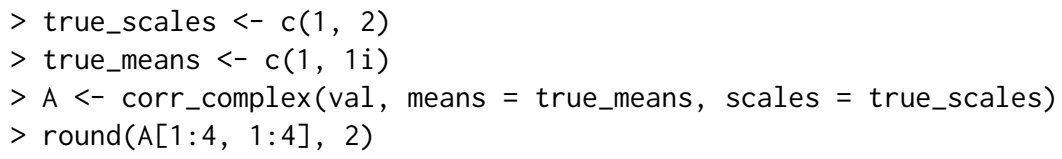

$$
[, 1] \quad[, 2] \quad[, 3] \quad[, 4]
$$

$[1] \quad 1.00+,0.00 \mathrm{i} \quad 0.59-0.27 \mathrm{i} \quad 0.25-0.10 \mathrm{i} \quad 0.89+0.11 \mathrm{i}$

$[2] \quad 0.59+,0.27 i \quad 1.00+0.00 i \quad 0.20+0.00 i \quad 0.42+0.26 i$

$[3] \quad 0.25+,0.10 \mathrm{i} \quad 0.20+0.00 \mathrm{i} \quad 1.00+0.00 \mathrm{i} \quad 0.10+0.06 \mathrm{i}$

$[4] \quad 0.89-,0.11 \mathrm{i} \quad 0.42-0.26 \mathrm{i} \quad 0.10-0.06 \mathrm{i} \quad 1.00+0.00 \mathrm{i}$

Function corr_complex () is a complex generalization of $\operatorname{corr}()$; matrix A is Hermitian positivedefinite:

$>\operatorname{all}($ eigen $(A) \$$ values $>0)$

[1] TRUE

It is now possible to make a single multivariate observation $d$ of this process, using $\beta=(1,1+i, 1-2 i)^{T}$ :

$>$ true_beta $<-c(1,1+1 i, 1-2 i)$

$>d<-\operatorname{drop}(\operatorname{rcmvnorm}(n=1$, mean $=$ regressor.multi $(v a l) \% * \%$ true_beta, sigma $=A)$ )

$>\operatorname{head}(d)$

[1] $3.212719+1.594901 \mathrm{i} 1.874278+0.345517 \mathrm{i} 3.008503-0.767618 \mathrm{i} 3.766526+2.071882 \mathrm{i}$

[5] $3.712913+0.800983 i \quad 3.944167+0.924833 i$ 
Thus $d$ is a single observation from a complex multivariate Gaussian distribution. Most of the functions of the emulator package operate without modification. Thus betahat. fun(), which calculates the maximum likelihood estimate $\hat{\boldsymbol{\beta}}=\left(H^{*} A^{-1} H\right)^{-1} H^{*} A^{-1} \mathbf{y}$ takes complex values directly:

$>$ betahat.fun(val, $\operatorname{solve}(A), d)$

const a b

$0.593632-0.0128655 i \quad 0.843608+1.0920437$ i $1.140372-2.5053751 i$

However, because the likelihood function is different, the interpolant () functionality is implemented in the cmvnorm package by interpolant.quick. complex(), named in analogy to function interpolant.quick() of package emulator.

For example, it is possible to evaluate the posterior distribution of the process at $(0.5,0.3+0.1 i)$, a point at which no observation has been made:

$>$ interpolant.quick.complex(rbind $(c(0.5,0.3+0.1 i)), d$, val, solve(A),

+ scales $=$ true_scales, means $=$ true_means, give. $Z=$ TRUE $)$

\$mstar.star

[1] 1.706402-1.008601i

$\$ Z$

[1] 0.203295

\$prior

[1] $1.608085-0.104419 i$

Thus the posterior distribution for the process is complex Gaussian at this point with a mean of about $1.71-1.01 i$ and a variance of about 0.2 .

\section{Analytic functions}

These techniques are now used to emulate an analytic function of several complex variables. A complex function's being analytic is a very strong restriction; Needham (2004) uses 'rigidity' to describe the severe constraint that analyticity represents.

Here the Weierstrass $\sigma$-function (Chandrasekharan, 1985) is chosen as an example, on the grounds that Littlewood and Offord (1948) consider it to be a typical entire function in a well-defined sense. The elliptic package (Hankin, 2006) is used for numerical evaluation.

The $\sigma$-function takes a primary argument $z$ and two invariants $g_{1}, g_{2}$, so a three-column complex design matrix is required:

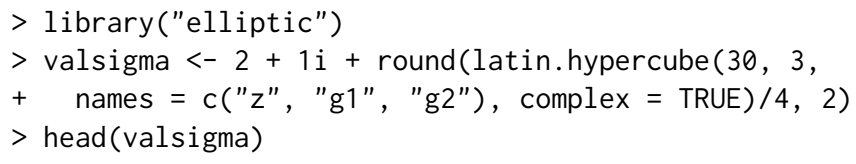

$[1] 2.17+,1.15 i \quad 2.09+1.22 i \quad 2.21+1.09 i$

$[2] 2.11+,1.01 \mathrm{i} 2.04+1.03 \mathrm{i} 2.25+1.15 \mathrm{i}$

$[3] 2.10+,1.04 \mathrm{i} 2.15+1.00 \mathrm{i} 2.22+1.20 \mathrm{i}$

$[4] 2.13+,1.10 \mathrm{i} 2.24+1.21 \mathrm{i} 2.01+1.16 \mathrm{i}$

$[5] 2.20+,1.00 \mathrm{i} 2.20+1.06 \mathrm{i} 2.08+1.08 \mathrm{i}$

$[6] 2.05+,1.10 \mathrm{i} 2.19+1.04 \mathrm{i} 2.11+1.03 i$

(an offset is needed because $\sigma\left(z, g_{1}, g_{2}\right)=z+\mathcal{O}\left(z^{5}\right)$ ). The $\sigma$-function can now be evaluated at the points of the design matrix:

$>$ dsigma <- apply(valsigma, 1, function(u) sigma(u[1], g = u[2:3]))

One way of estimating the roughness parameters is to use maximum likelihood. The likelihood for any set of roughness parameters is given by Oakley (1999) as $\left(\sigma^{2}\right)^{-\frac{n-q}{2}}|A|^{-1 / 2}\left|H^{T} A^{-1} H\right|^{-1 / 2}$ with complex generalization $\left(\sigma^{2}\right)^{-(n-q)}|A|^{-1}\left|H^{*} A^{-1} H\right|^{-1}$ which is calculated in the package by function scales.likelihood. complex(); this can be used to return the log-likelihood for a specific set of roughness parameters: 


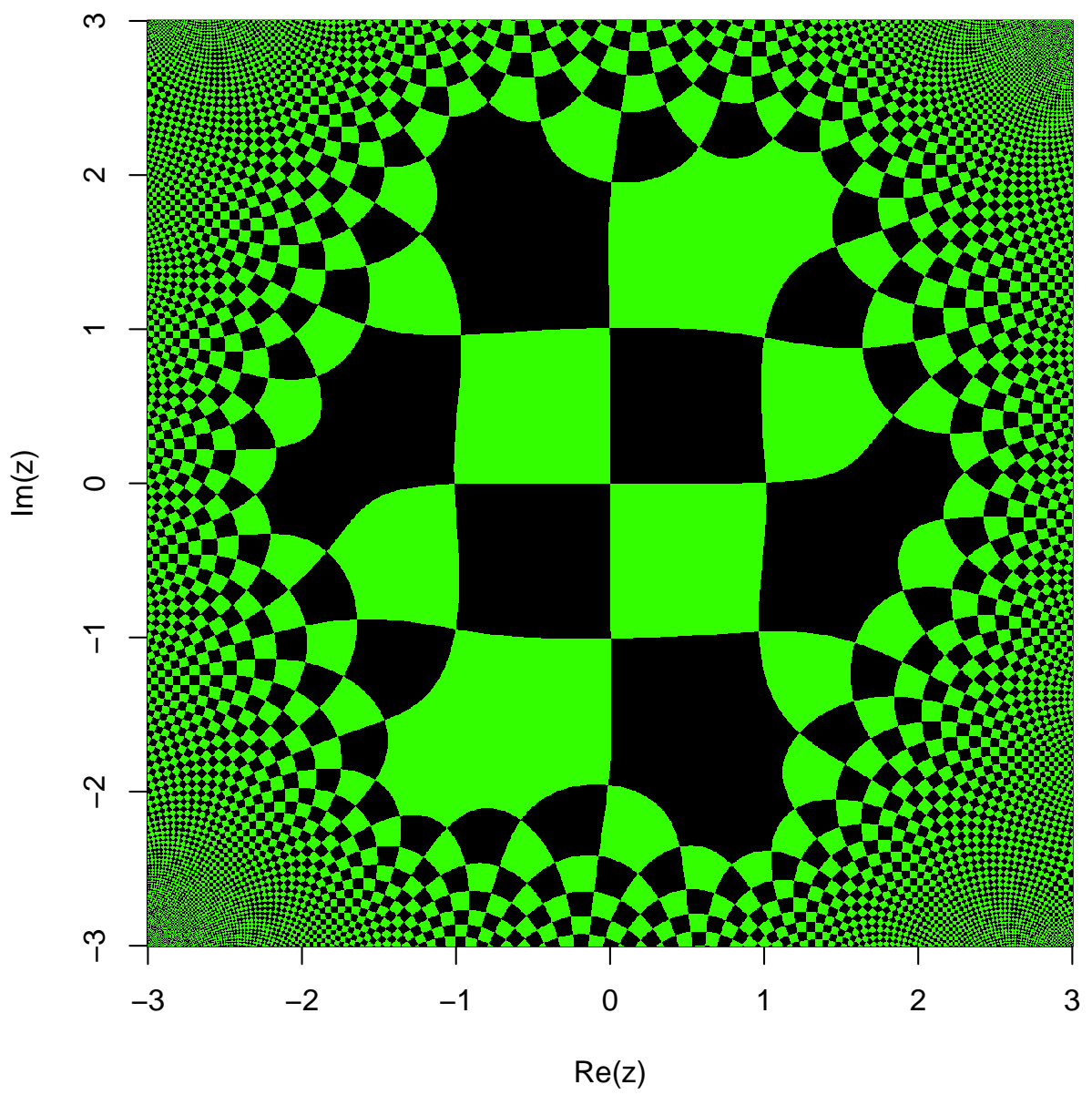

Figure 1: Visualization of the Weierstrass $\sigma$-function, specifically $\sigma(z ; 2+i, 2.2+1.1 i)$ in the region of the complex plane $-4 \leqslant \operatorname{Re}(z), \operatorname{Im}(z) \leqslant+4$; visualization is scheme 13 of Hankin (2006).

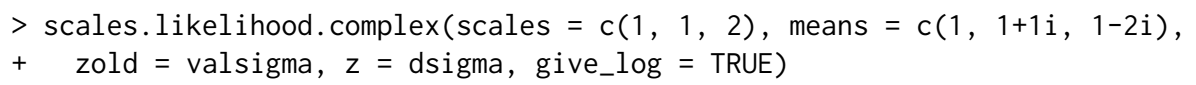

\section{[1] 144.5415}

Numerical methods can then be used to find the maximum likelihood estimate. Because function optim() optimizes over $\mathbb{R}^{n}$, helper functions are again needed which translate from the optimand to scales and means:

$>$ scales <- function $(x) \exp (x[c(1,2,2)])$
$>$ means <- function $(x) \times[c(3,4,4)]+1 i * x[c(5,6,6)]$

Because the diagonal elements of $\mathfrak{B}$ are strictly positive, their logarithms are optimized, following Hankin (2005); it is implicitly assumed that the scales and means associated with $g_{1}$ and $g_{2}$ are equal.

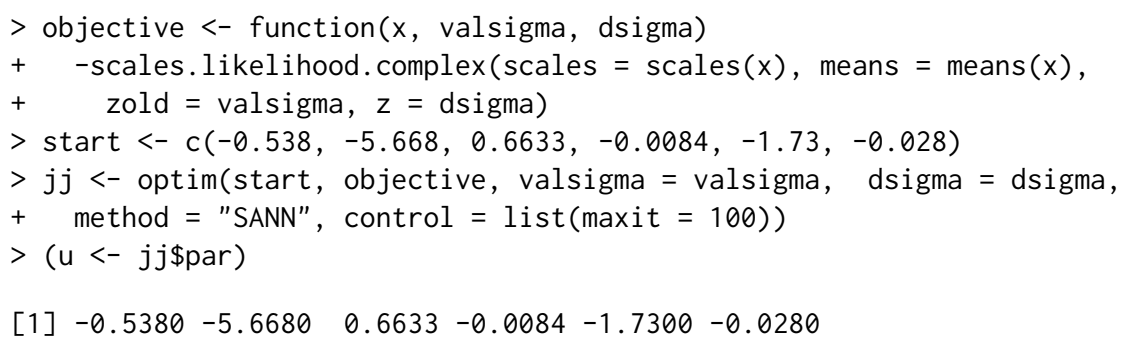

Function corr_complex() may now be used to calculate the covariance of the observations:

$>$ Asigma $<-\operatorname{cor} r_{-} \operatorname{complex}(z 1=$ valsigma, $\operatorname{scales}=\operatorname{scales}(u)$, means $=\operatorname{means}(u))$

So now we can compare the emulator against the "true" value: 


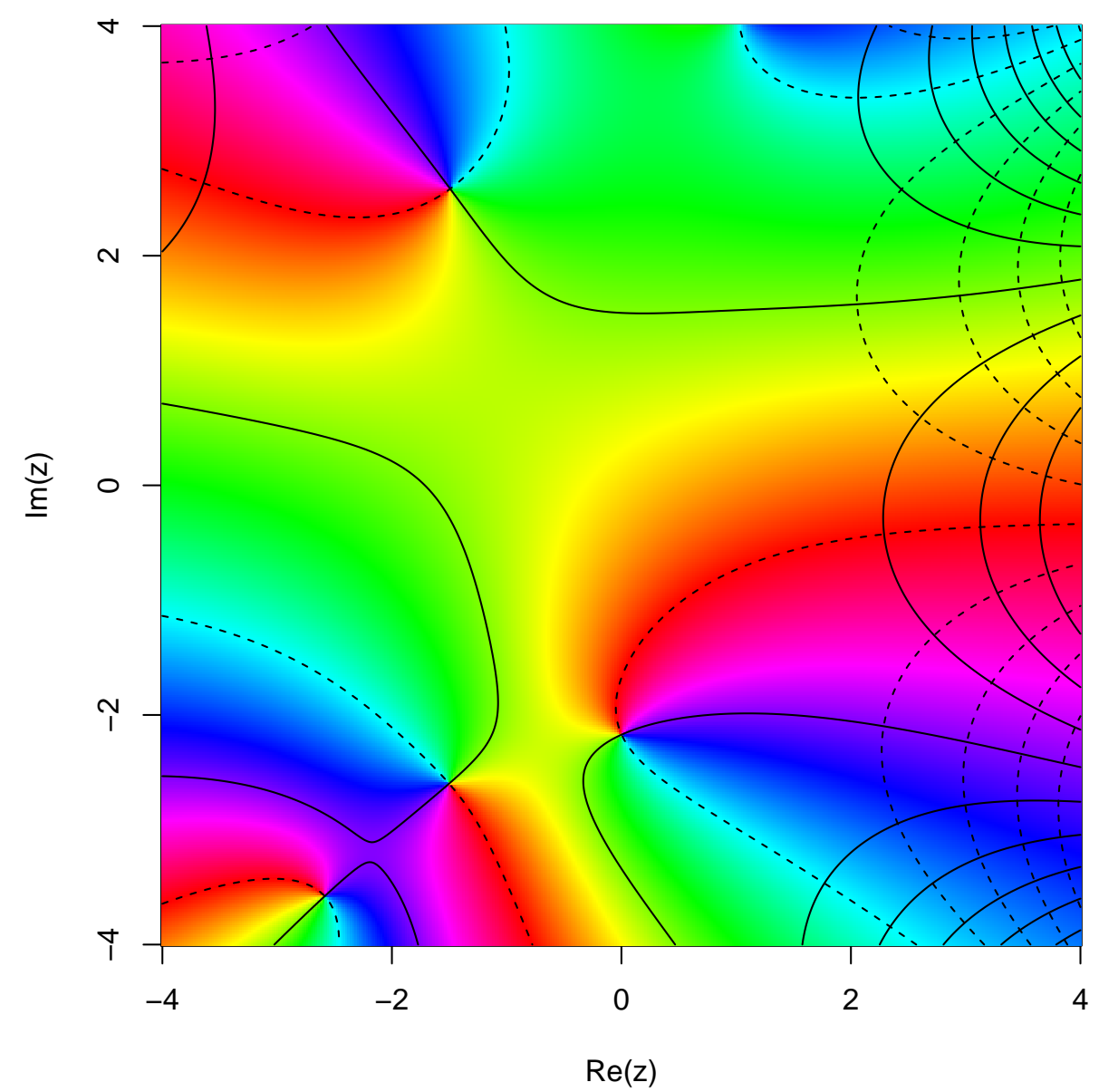

Figure 2: Visualization of the Weierstrass $\sigma$-function, specifically $\sigma\left(6+1 i ; g_{1}=z, g_{2}=1\right)$ in the region of the complex plane $-4 \leqslant \operatorname{Re}(z), \operatorname{Im}(z) \leqslant+4$; visualization is scheme 8 of Hankin (2006).

$>$ interpolant.quick. complex $(\operatorname{rbind}(\mathrm{c}(2+1 \mathrm{i}, 2+1 \mathrm{i}, 2+1 \mathrm{i}))$, zold $=$ valsigma,

$+d=$ dsigma, Ainv $=\operatorname{solve}($ Asigma $), \operatorname{scales}=\operatorname{scales}(u)$, means $=$ means $(u))$

[1] $3.078956+1.259993 i$

$>\operatorname{sigma}(2+1 i, g=c(2+1 i, 2+1 i))$

[1] $3.078255+1.257819 \mathrm{i}$

showing reasonable agreement. It is also possible to test the hypothesis $H_{\mathbb{R}}: \mathbf{m} \in \mathbb{R}^{2}$ (that is, the variance matrix $A$ is real), by calculating the likelihood ratio of the unconstrained model (6) to that obtained by $H_{\mathbb{R}}$. This may be achieved by constraining the optimization to satisfy $\mathbf{m} \in \mathbb{R}^{2}$ :

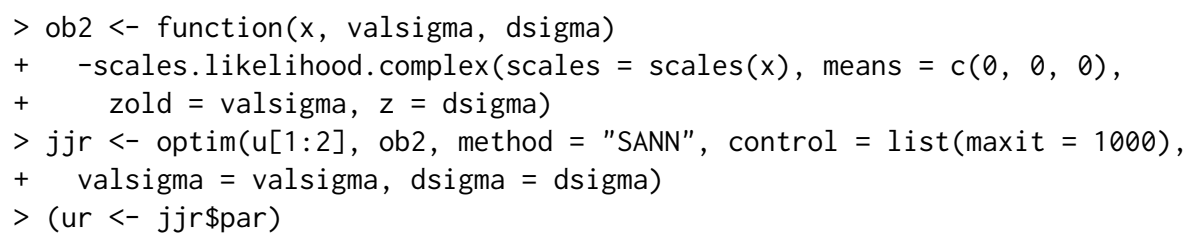

[1] $0.2136577-4.2640825$

so the test statistic $D$ is given by

$>$ LR <- scales.likelihood. complex (scales $=\operatorname{scales}($ ur $)$, means $=c(\theta, 0,0)$,

+ zold = valsigma, $z=$ dsigma $)$

$>$ LC <- scales.likelihood. complex $($ scales $=\operatorname{scales}(u)$, means $=$ means $(u)$,

+ zold = valsigma, $\mathrm{z}=$ dsigma $)$

$>(D<-2 *(L C-L R))$ 
[1] 22.17611

Observing that $D$ is in the tail region of its asymptotic distribution, $\chi_{3}^{2}$, the hypothesis $H_{\mathbb{R}}$ may be rejected.

\section{Conclusions}

The cmvnorm package for the complex multivariate Gaussian distribution has been introduced and motivated. The Gaussian process has been generalized to the complex case, and a complex generalization of the emulator technique has been applied to an analytic function of several complex variables. The complex variance matrix was specified using a novel parameterization which accommodated non-real covariances in the context of circulary symmetric random variables. Further work might include numerical support for the complex multivariate Student $t$ distribution.

\section{Bibliography}

K. Chandrasekharan. Elliptic Functions. Springer-Verlag, 1985. [p77]

W. Feller. An Introduction to Probability Theory and its Applications, volume 2. Wiley, 1971. [p74]

A. Genz, F. Bretz, T. Miwa, X. Mi, F. Leisch, F. Scheipl, and T. Hothorn. motnorm: Multivariate Normal and $t$ Distributions, 2014. URL http://CRAN. R-project.org/package=mvtnorm. R package version 1.0-0. [p73]

N. R. Goodman. Statistical analysis based on a certain multivariate complex Gaussian distribution (an introduction). The Annals of Mathematical Statistics, 34(1):152-177, Mar. 1963. [p73]

R. K. S. Hankin. Introducing BACCO, an R bundle for Bayesian analysis of computer code output. Journal of Statistical Software, 14(16):1-20, Oct. 2005. [p73, 78]

R. K. S. Hankin. Introducing elliptic, an R package for elliptic and modular functions. Journal of Statistical Software, 15(7):1-22, Feb. 2006. [p77, 78, 79]

R. K. S. Hankin. Introducing multivator: A multivariate emulator. Journal of Statistical Software, 46(8): 1-20, Feb. 2012. [p75]

R. K. S. Hankin. cmonorm: The Complex Multivariate Gaussian Distribution, 2015. URL http: //CRAN. Rproject. org / package =cmvnorm. R package version 1.0-2. [p73]

S. Kay. Modern Spectral Analysis. Prentice-Hall, 1989. [p73]

S. G. Krantz. What is several complex variables? The American Mathematical Monthly, 94(3):236-256, Mar. 1987. [p76]

J. E. Littlewood and A. C. Offord. On the distribution of zeros and $a$-values of a random integral function (II). The Annals of Mathematics, 49(4):885-952, Oct. 1948. [p77]

D. P. Mandic, S. Javidi, S. L. Goh, A. Kuh, and K. Aihara. Complex-valued prediction of wind profile using augmented complex statistics. Renewable Energy, 34:196-201, 2009. [p73]

T. Needham. Visual Complex Analysis. Clarendon Press, Oxford, 2004. [p77]

J. Oakley. Bayesian Uncertainty Analysis for Complex Computer Codes. PhD thesis, University of Sheffield, 1999. [p74, 75, 77]

L. H. Ozarow. Information theoretic considerations for cellular mobile radio. IEEE Transactions on Vehicular Technology, 43(2):359-378, May 1994. [p73]

Robin K. S. Hankin

Auckland University of Technology

2-14 Wakefield Street

Auckland NZ

hankin.robin@gmail.com 